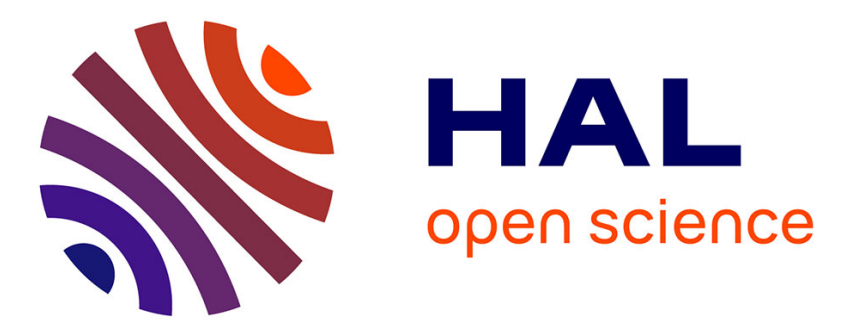

\title{
Thermoelectric properties and stability of nanostructured chromium disilicide CrSi 2
}

M. Khalil, Adrien Moll, M. Godfroy, A. Letrouit-Lebranchu, B. Villeroy, E. Alleno, Romain Viennois, Mickael Beaudhuin

\section{- To cite this version:}

M. Khalil, Adrien Moll, M. Godfroy, A. Letrouit-Lebranchu, B. Villeroy, et al.. Thermoelectric properties and stability of nanostructured chromium disilicide CrSi 2. Journal of Applied Physics, 2019, 126 (13), pp.135103. 10.1063/1.5117152 . hal-02338762

\section{HAL Id: hal-02338762 \\ https://hal.umontpellier.fr/hal-02338762}

Submitted on 30 Oct 2019

HAL is a multi-disciplinary open access archive for the deposit and dissemination of scientific research documents, whether they are published or not. The documents may come from teaching and research institutions in France or abroad, or from public or private research centers.
L'archive ouverte pluridisciplinaire HAL, est destinée au dépôt et à la diffusion de documents scientifiques de niveau recherche, publiés ou non, émanant des établissements d'enseignement et de recherche français ou étrangers, des laboratoires publics ou privés. 


\section{Thermoelectric properties and stability of nanostructured chromium disilicide $\mathrm{CrSi}_{2}$}

Cite as: J. Appl. Phys. 126, 135103 (2019); https://doi.org/10.1063/1.5117152

Submitted: 02 July 2019 . Accepted: 10 September 2019 . Published Online: 01 October 2019

M. Khalil, A. Moll, M. Godfroy, A. Letrouit-Lebranchu, B. Villeroy, E. Alleno, R. Viennois (D), and M.

Beaudhuin (D)

\section{COLLECTIONS}

Paper published as part of the special topic on Advanced Thermoelectrics

Note: This paper is part of the special topic on Advanced Thermoelectrics.
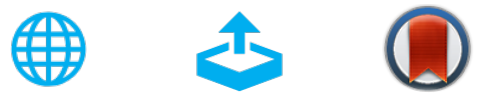

\section{ARTICLES YOU MAY BE INTERESTED IN}

Dynamic behavior of helium bubbles at high temperature in Si studied by in situ TEM, STEM-

EELS, and TDS

Journal of Applied Physics 126, 135104 (2019); https://doi.org/10.1063/1.5118684

Ferroelectric ordering and energy storage capacity in lead-free $\mathrm{Ba}\left(\mathrm{Zr}_{0.2} \mathrm{Ti}_{0.8}\right) \mathrm{O}_{3}$ nanoscale film capacitors fabricated using pulsed laser deposition technique

Journal of Applied Physics 126, 134101 (2019); https://doi.org/10.1063/1.5117170

Magnetic and cryogenic magnetocaloric properties of $\mathrm{NaGdF}_{4}$ nanocrystals

Journal of Applied Physics 126, 135101 (2019); https://doi.org/10.1063/1.5114993

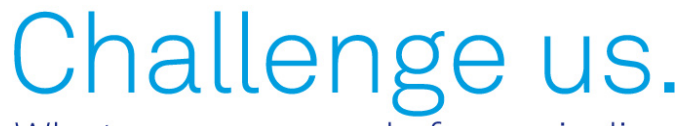

What are your needs for periodic signal detection?

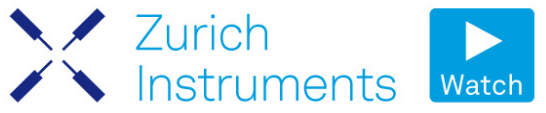

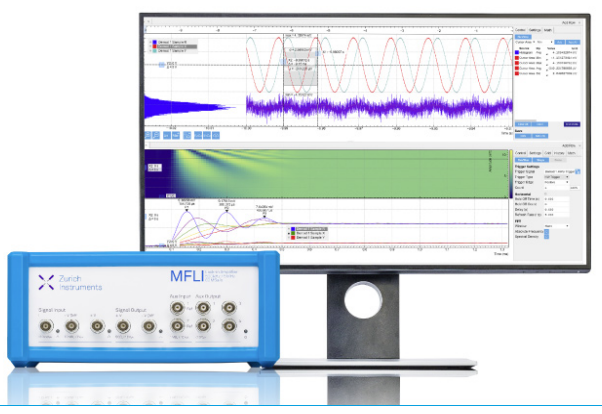

126, 135103 


\title{
Thermoelectric properties and stability of nanostructured chromium disilicide $\mathrm{CrSi}_{2}$
}

Cite as: J. Appl. Phys. 126, 135103 (2019); doi: 10.1063/1.5117152

Submitted: 2 July 2019 - Accepted: 10 September 2019 . Published Online: 1 October 2019

M. Khalil, ${ }^{7}$ A. Moll, ${ }^{1}$ M. Godfroy, ${ }^{7}$ A. Letrouit-Lebranchu, ${ }^{1}$ B. Villeroy, ${ }^{2}$ E. Alleno, ${ }^{2}$ R. Viennois, ${ }^{1}$ (D) and M. Beaudhuin ${ }^{1, a)}$ (iD AFFILIATIONS

${ }^{7}$ ICGM, University of Montpellier, CNRS, ENSCM, Montpellier 34095, France

${ }^{2}$ ICMPE-CMTR, CNRS UMR 7182, 2-8, rue H. Dunant, Thiais 94320, France

Note: This paper is part of the special topic on Advanced Thermoelectrics.

${ }^{a)}$ Author to whom correspondence should be addressed: mickael.beaudhuin@umontpellier.fr

\begin{abstract}
$\mathrm{CrSi}_{2}$ alloy presents a strong interest for thermoelectric applications; however, its thermal conductivity is still too high and limits strongly its figure of merit. By combining experiment and modeling, we show that the nanostructuring of $\mathrm{CrSi}_{2}$ leads to a strong decrease in the thermal conductivity without affecting much the electronic transport properties. The thermal conductivity of nanostructured $\mathrm{CrSi}_{2}$ $(\sim 45 \mathrm{~nm})$ has also been determined as a function of the density. We predict that it would be about $5.5 \mathrm{~W} / \mathrm{mK}$ at RT for a $100 \%$ dense sample, i.e., twice smaller than in bulk samples. We also give for the first time, a prediction of the effect of alloying on the thermal conductivity using the Callaway-Klemens model. To consider final applications, it is necessary to also investigate the thermal stability of nanostructured compounds. We show that grain coarsening of nanostructured $\mathrm{CrSi}_{2}$, even if it remains limited, happens above $1073 \mathrm{~K}$ and will deserve further attention.
\end{abstract}

Published under license by AIP Publishing. https://doi.org/10.1063/1.5117152

\section{INTRODUCTION}

The interest in renewable energies to sustain economic growth and environmental concerns has increased dramatically these last few decades. Among renewable energies, thermoelectric materials, which allow one to convert heat into electricity, without moving part, can participate in this effort. Their thermoelectric performances are characterized by their dimensionless figure of merit ZT, such as

$$
Z T=\frac{S^{2} \sigma}{\kappa} T
$$

with $S$ being the Seebeck coefficient $\left(\mathrm{V} \mathrm{K}^{-1}\right), T$ being the absolute temperature (Kelvin), $\sigma$ being the electrical conductivity $\left(\mathrm{S} \mathrm{m}^{-1}\right)$, and $\kappa$ being the thermal conductivity $\left(\mathrm{W} \mathrm{m}^{-1} \mathrm{~K}^{-1}\right)$.

There are different ways to improve the figure of merit, either by increasing $S^{2} \sigma$ (also known as the power factor PF) or by decreasing $\kappa$.

In this work, we study a semiconducting silicide material that presents a strong interest for thermoelectric applications, namely, $\mathrm{CrSi}_{2}$ (space group $\mathrm{P}_{2} 22$ ). ${ }^{1}$ This compound has a high creep resistance and a good oxidation resistance, which makes it suitable for high temperature applications. ${ }^{2}$ It is a highly degenerated $p$-type semiconductor with a narrow indirect bandgap of $0.35 \mathrm{eV}^{3,4}$ It has a relatively high Seebeck coefficient $\left(S \sim 100 \mu \mathrm{V} \mathrm{K}^{-1}\right.$ at RT), ${ }^{2,5}$ and a relatively high electrical conductivity $\left(\sigma \sim 1000 \mathrm{~S} \mathrm{~m}^{-1}\right)$. Unfortunately, its thermal conductivity is rather high $\left(\kappa \sim 10 \mathrm{~W} \mathrm{~m}^{-1} \mathrm{~K}^{-1}\right.$ at RT), which is detrimental to the thermoelectric performances. It is well known that $\kappa$ is the sum of two contributions: $\kappa_{\mathrm{e}}$, which describes the electronic thermal transport and $\kappa_{\mathrm{l}}$, which describes the lattice thermal transport. It is not possible to decrease $\kappa_{\mathrm{e}}$ without decreasing $\sigma$. However, as $\kappa_{1}$ represents about $93 \%$ of the total thermal conductivity $\kappa$ in pure $\mathrm{CrSi}_{2}$, ${ }^{7,8}$ there is consequently a strong potential to optimize this material by decreasing $\kappa_{1}$. Different research routes can be considered, the first one is the alloying: a distortion of the unit cell can be obtained by substituting an element on the lattice of the compound, which creates strains and mass fluctuations that can scatter phonons; this effect has been efficiently demonstrated in $\mathrm{Mg}_{2} \mathrm{Si}_{1-\mathrm{x}} \mathrm{Sn}_{\mathrm{x}}$ compounds in Ref. 9. The second one concerns the nanostructuration as it is also strongly favorable to decrease $\kappa_{1}$ by creating multiple interfaces that scatter the phonons of various mean free paths. ${ }^{10,11}$ We have previously 
shown that the thermal conductivity of $\mathrm{CrSi}_{2}$ samples can be decreased by a factor of $\sim 10$ close to $1 \mathrm{~W} \mathrm{~m}^{-1} \mathrm{~K}^{-1}$ by combining both nanostructuration and porosity. ${ }^{10}$ However, the electrical resistivity was increased as well by a factor of $\sim 10$. Both effects are related to significant porosity in the studied samples. Recently, we have also shown the importance of reducing the porosity for approaching bulk values of the electrical resistivity, whereas the influence of the porosity on the thermopower was negligible. ${ }^{12}$ Nevertheless, a similar study for the porosity dependence of the thermal conductivity of nanostructured $\mathrm{CrSi}_{2}$ samples is still lacking, and there is still no study on the impact of the nanostructuration on fully dense $\mathrm{CrSi}_{2}$ samples. Up to now, the maximum $\mathrm{ZT}$ value of pure $\mathrm{CrSi}_{2}$ is 0.25 at $900 \mathrm{~K}$ in single crystal along the c-axis. ${ }^{13,14}$

A better understanding of the phonon scattering mechanisms is the first step of a research path to decrease the lattice component of the thermal conductivity of $\mathrm{CrSi}_{2}$, an indispensable action before its use in thermoelectric applications.

In this paper, we first present a stability study $(300 \mathrm{~K}-1500 \mathrm{~K})$ of the $\mathrm{CrSi}_{2}$ nanostructured samples, which is a prerequisite for evaluating the ability of nanostructured samples to be used for long duration-high temperature applications. Then, for the first time, we report moderate temperature measurements $(300 \mathrm{~K}-800 \mathrm{~K})$ of the thermal conductivity for two nanostructured samples with similar nanocrystallite size but with differing porosity and give a prediction for $100 \%$ dense nanostructured samples. Finally, we analyze the impact of the porosity, of the small grain size, and of other phonon scattering mechanisms on the lattice thermal conductivity of $\mathrm{CrSi}_{2}$.

\section{MATERIALS AND METHODS}

\section{A. Sample preparations}

$\mathrm{CrSi}_{2}$ was synthesized by arc melting the elements in the stoichiometric ratio under the argon atmosphere using high purity $\mathrm{Cr}$ granules (99.995\% Alfa Aesar) and Si lumps (99.999\% Alfa Aesar). The ingots obtained by this technique were first crushed in an agate mortar and then milled in a Fritsch "Pulverisette 7" planetary micromill in order to decrease the crystallite size. A silicon nitride container $(45 \mathrm{ml})$ and five $15 \mathrm{~mm}$ diameter balls were used as the milling media with a ball to powder mass ratio fixed to 10:1. The speed of the supporting disc and of the grinding bowl was $575 \mathrm{rpm}$ and $1150 \mathrm{RPM}$, respectively, for all the experiments (ball acceleration $\sim 100 \mathrm{~m} / \mathrm{s}^{2}$ ). The container was sealed under the argon atmosphere in order to avoid contamination during the milling.

Spark Plasma Sintering (SPS) technique was used to prepare dense pellets using a "Dr. Sinter 515S Syntex" machine. Carbon dies with $8 \mathrm{~mm}$ and $10 \mathrm{~mm}$ diameter $(100 \mathrm{MPa})$ and a tungsten carbide die with an $8 \mathrm{~mm}$ diameter $(300 \mathrm{MPa})$ were used for these experiments along with a graphite foil as a protective layer between the samples and the die. Uniaxial pressure and DC pulses were both delivered by carbon or tungsten carbide punches at both sides.

\section{B. Sample characterization}

The samples were analyzed after each arc melting synthesis, ball milling, and sintering by using an X-Ray Diffraction apparatus (Philips X'PERT, Cu-K $\alpha$ radiation $1.5406 \AA$ with an accelerated detector PW3050/60 at $45 \mathrm{kV}$ and $30 \mathrm{~mA}$ settings). The Rietveld refinement with Fullprof software was used for structural analysis of each sample. The crystallite size was determined by using the microstructural analysis method from Fullprof software. ${ }^{15}$ The chemical homogeneity and composition of the samples were checked using an Energy Dispersive Analyser of X-Ray (EDX) Hitachi S4800 mounted on a Hitachi S2600N scanning electron microscope. Samples porosity was determined by measuring both the mass and the volume of the pellet and by comparing it to the theoretical density obtained by the Rietveld refinement. The stability of nanostructured $\mathrm{CrSi}_{2}$ was investigated using X-Ray Diffraction from $300 \mathrm{~K}$ to $1273 \mathrm{~K}$ on a Ta hot-plate under vacuum with $2 \mathrm{~h}$ dwell time for the analysis. Powders, dispersed on carbon films, were analyzed on a JEOL 2200 High Resolution Transmission Electron Microscope (HRTEM) at $200 \mathrm{kV}$, equipped with a CCD camera (GATAN USC) with $4092 \times 4092$ pixel $^{2}$.

The thermal conductivity $\kappa$ was derived from the thermal diffusivity $\alpha$ measured by a laser flash method using a Netzsch LFA 427 system, the specific heat $C_{p}$ from the data of Chen et al., ${ }^{16}$ and the theoretical density $d$ using the relationship $\kappa=\alpha \cdot C_{p} \cdot d$. The uncertainty on the measurement for the thermal conductivity is $10 \% .{ }^{17}$ The density of the bulk samples was determined by measuring both the mass and the volume of the pellet and by comparing to the theoretical density obtained by the Rietveld refinement. Electrical resistivity was characterized using a homemade apparatus by the Van der Pauw technique (more details are given in the supplementary material). The sample dimensions for electrical resistivity and thermal diffusivity measurements are $8 \mathrm{~mm} \emptyset \times 2 \mathrm{~mm}$ thickness.

\section{RESULTS AND DISCUSSION}

\section{A. Size of $\mathrm{CrSi}_{2}$ nanocrystallites}

After arc melting and ball milling of the samples, the Rietveld refinement shows that pure $\mathrm{CrSi}_{2}$ is obtained with the unit cell parameters $a=4.433(4) \AA$ and $c=6.366(4) \AA$, in good agreement with the literature $(a=4.442(8) \AA \text { and } c=6.366(8) \AA)^{10}$ (see, for instance, Fig. S1 in the supplementary material). As can be seen in Fig. 1, the analysis of the XRD patterns show that the crystallites size decreases from about $400 \mathrm{~nm}$ after $22 \mathrm{~min}$ of milling to about $17 \pm 2 \mathrm{~nm}$ after $24 \mathrm{~h}$ of milling. These results are confirmed by the SEM and the bright-field TEM images as shown in Fig. 2 with a typical size ranging from 6 to $11 \mathrm{~nm}$ for $4 \mathrm{~h}$ milling time. This milling time has been chosen for the whole study. This observation indicates that increasing the ball milling time does not further refine the grain size. One also notices the very strong increase of the internal microstrain from $\sim 0.45 \pm 0.04 \%$ after $22 \mathrm{~min}$ of milling up to $1.05 \pm 0.05 \%$ after $24 \mathrm{~h}$ of milling. This can be explained by the formation of defects during the highly energetic milling process; a similar behavior has been observed by several groups. ${ }^{18,19}$ The SEM images of these samples show that these nanoparticles are aggregated with typical size ranging from 0.5 to $1.5 \mu \mathrm{m}$. The determination of the crystallite size for shorter milling time by TEM suffer from both the difficulty to obtain good enough TEM images due to the large size of the crystallites and because they are in the form of thick aggregates. 


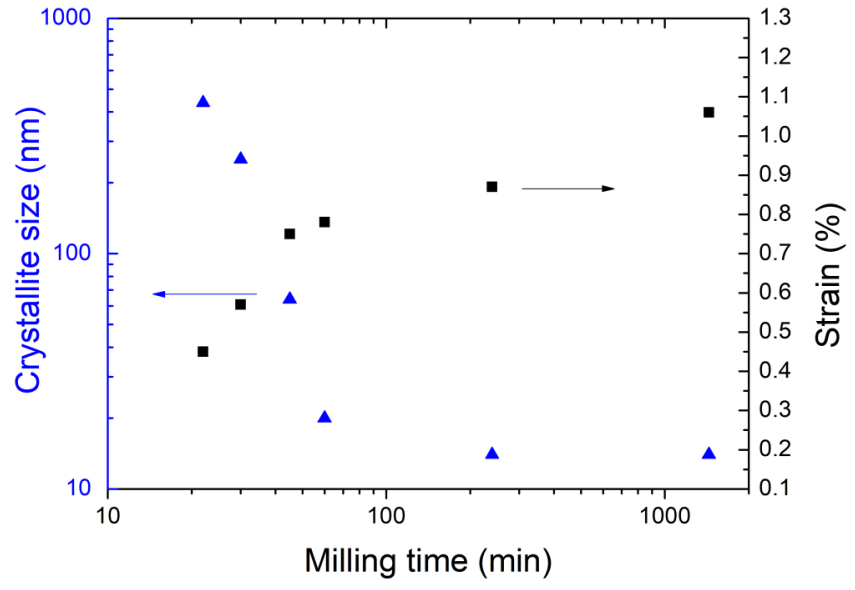

FIG. 1. Influence of the milling time on the microstrain and crystallite size of ball-milled $\mathrm{CrSi}_{2}$ samples.

\section{B. Thermal stability of $\mathrm{CrSi}_{2}$ nanopowders}

The stability of the $\mathrm{CrSi}_{2}$ nanocrystallites under powder form has been investigated as a function of temperature by an in situ XRD technique under vacuum. The powder patterns are displayed in Fig. 3. At $300 \mathrm{~K}$, the pattern is typical of a ball-milled powder with broad peaks. The noticeable background intensity at low angle is in turn characteristic of the occurrence of an(several) amorphous phase(s), formed at the milling stage. By increasing the temperature, a decrease of the full width at half maximum is observed, which means that the crystallites are coarsening. The displacement of the peaks toward lower angles is due to the thermal expansion of the sample. In parallel, one can note that the background is flattened at $973 \mathrm{~K}$, which denotes the crystallization of the amorphous phase (s). Along with the increase of temperature and sample crystallinity, secondary phases are observed above $1073 \mathrm{~K}: \mathrm{Cr}_{5} \mathrm{Si}_{3}, \mathrm{CrSi}$, and $\mathrm{Si}$ were identified. These phases could likely be crystallized from the amorphous phase(s). Since the crystalline phases exhibit the Si concentration of both larger and smaller than $\mathrm{CrSi}_{2}$, either a single amorphous phase decomposes peritectically or several amorphous phases crystallize without changing their composition.
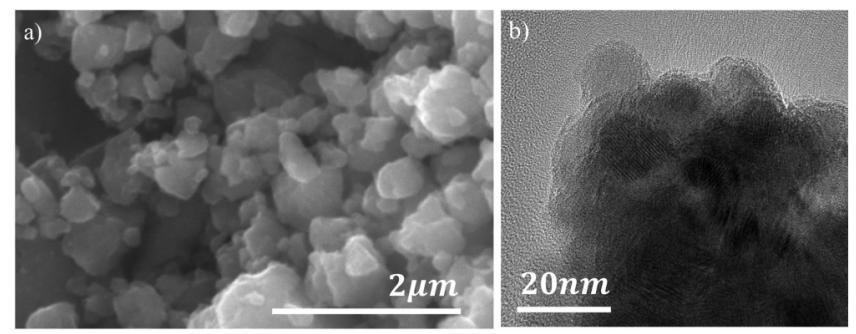

FIG. 2. (a) SEM image of $\mathrm{CrSi}_{2}$ aggregates in the $4 \mathrm{~h}$ ball-milled sample, (b) the bright-field HRTEM image of $\mathrm{CrSi}_{2}$ crystallite in the same sample.

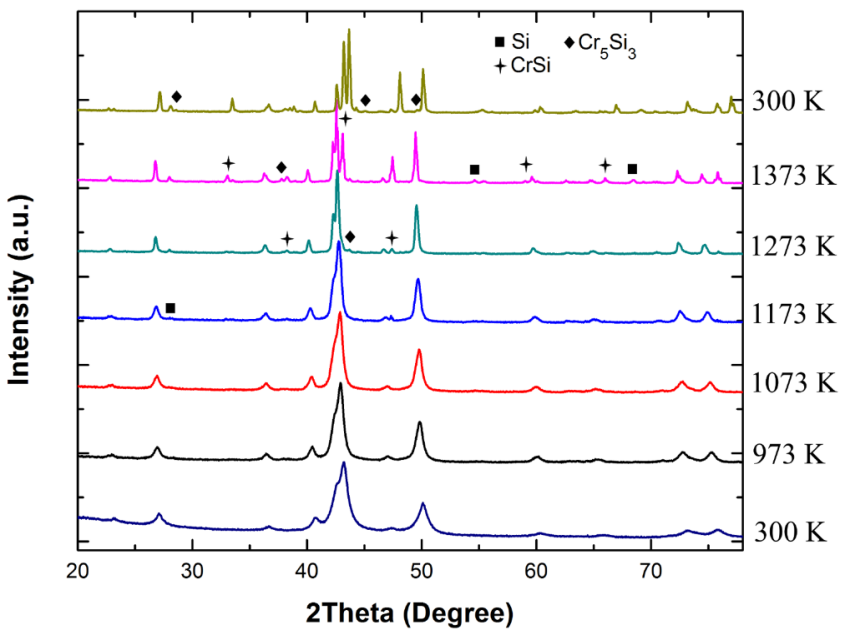

FIG. 3. In situ $\mathrm{X}$-ray patterns of $\mathrm{CrSi}_{2}$ nanopowders as a function of temperature.

In Fig. 4, we provide the microstructural analysis of the XRD patterns from Fig. 3. The decrease of the line width corresponds to a small increase in the crystallite size from about $10 \mathrm{~nm}$ at $300 \mathrm{~K}$ to about $20 \mathrm{~nm}$ at $1073 \mathrm{~K}$, followed by a strong increase up to $\sim 120 \mathrm{~nm}$ at $1373 \mathrm{~K}$. In parallel and as it could be expected, the microstrain is smoothly released by the annealing. This leads to a decrease of the residual strain from about $0.5 \pm 0.05 \%$ at $300 \mathrm{~K}$ to about $0.05 \pm 0.05 \%$ at $1373 \mathrm{~K}$. Neither the kinetics of the strain release nor of the crystallite coarsening was studied in this work.

To summarize, we observe that the microstructure of $\mathrm{CrSi}_{2}$ nanopowders is fairly stable up to $1073 \mathrm{~K}$ and that grain coarsening occurs only beyond this temperature.

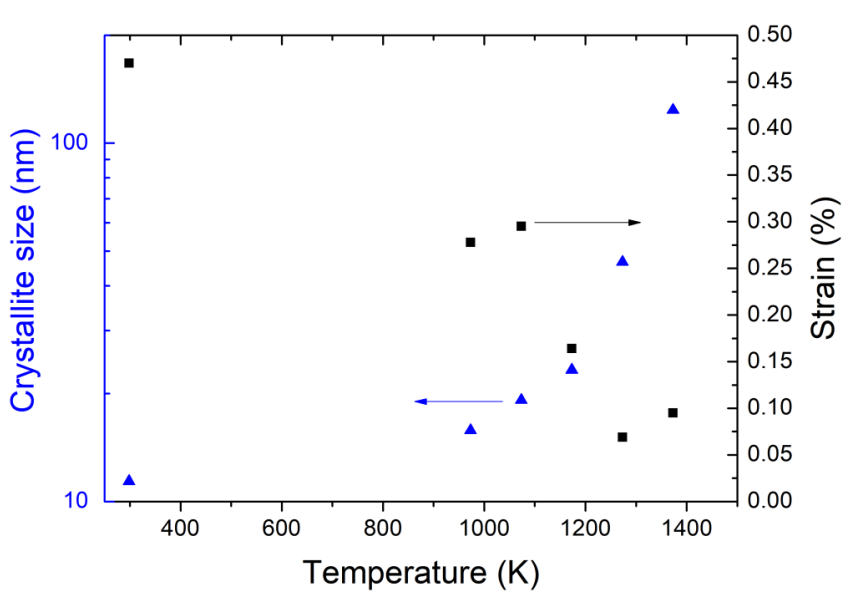

FIG. 4. Microstructural analysis of the powder XRD patterns displayed in Fig. 3 : crystallite size and microstrain, as a function of temperature. 
TABLE I. Influence of the sintering conditions on the porosity and on the crystallite size of $\mathrm{CrSi}_{2}$

\begin{tabular}{lccc}
\hline \hline Temperature (K) & 1073 & 1073 & 1173 \\
Pressure (MPa) & 100 & 300 & 100 \\
Density (\%) & 74 & 82 & 94 \\
Crystallite size (nm) & $\sim 45$ & $\sim 45$ & $\sim 45$ \\
\hline \hline
\end{tabular}

\section{MICROSTRUCTURE AND THERMAL CONDUCTIVITY IN DENSIFIED $\mathrm{CrSi}_{2}$}

\section{A. Crystallite size and density of SPS pellets}

We have investigated the effect of the temperature and pressure on the sintering of nanostructured $\mathrm{CrSi}_{2}$ in order to maintain the nanometer size of the crystallites. The optimized SPS conditions are given in Table I. With the condition $\mathrm{T}=1073$ or $1173 \mathrm{~K}$ and $\mathrm{P}=100$ or $300 \mathrm{MPa}$, it has been observed that the crystallite size is slightly the same whatever the sintering temperature or pressure is. The highest density $(>94 \%)$ has been obtained at $1173 \mathrm{~K}$ with a pressure of $100 \mathrm{MPa}$ and a crystallite size of $\sim 45 \mathrm{~nm}$. There is consequently an increase of the crystallize size by a factor of about 3 after SPS (the patterns are displayed in Fig. S2 in the supplementary material).

\section{B. Thermal conductivity of $74 \%$ and $94 \% \mathrm{CrSi}_{2}$}

The thermal conductivity measurements have been performed on samples with $74 \%$ and $94 \%$ density and similar crystallite size (about $45 \mathrm{~nm}$ ), the results are shown in Fig. 5. It is observed in Fig. 5(a) that a strong decrease of the total thermal conductivity from $\sim 11 \mathrm{~W} / \mathrm{mK}$ at $300 \mathrm{~K}$ for bulk $\mathrm{CrSi}_{2}{ }^{2}$ to, respectively, $\sim 5$ and $\sim 3.5 \mathrm{~W} / \mathrm{mK}$ for nanostructured samples with, respectively, $94 \%$ and $74 \%$ density. At high temperatures, the thermal conductivity drops slightly from $\sim 6$ to, respectively, 4 and $3 \mathrm{~W} / \mathrm{mK}$. Whereas the minimum thermal conductivity is obtained at about $770 \mathrm{~K}$ for bulk $\mathrm{CrSi}_{2}$, for nanostructured ones, the minimum thermal conductivity decreases to lower temperature $(\sim 700 \mathrm{~K}$ and $\sim 650 \mathrm{~K}$ for, respectively, $94 \%$ and $74 \%$ density samples).

Electronic contribution to the thermal conductivity was calculated based on the electrical resistivity measurement given in Fig. S3 in the supplementary material, using Wiedemann's Franz Law $\kappa_{e}=L T / \rho$ with $L=2.4410^{-8} \mathrm{~W} \Omega \mathrm{K}^{-2},{ }^{13} \quad T$ being the temperature in Kelvin, and $\rho$ being the electrical resistivity in Ohm meters. For bulk $\mathrm{CrSi}_{2}$ characterized in Ref. 2 and for $94 \%$ dense samples, $\kappa_{e}$ increases around $700-750 \mathrm{~K}$, for the $74 \%$ dense samples, $\kappa_{e}$ is negligible and can be attributed to an increase of electron scattering. $\kappa_{e}$ weights, respectively, only $2 \%$ and $8 \%$ of $\kappa_{T}$ for $74 \%$ and $94 \%$ dense samples at $300 \mathrm{~K}$ and $7 \%$ and $17 \%$ at $750 \mathrm{~K}$. When looking to the different contributions to the total thermal conductivity in Fig. 5, one can conclude that the increase of the thermal conductivity at high temperature is due to the electronic contribution, which increases with the temperature.

Our above results explain also why we find a lower thermal conductivity than in hot-pressed nanostructured powders (between 7.7 and $11 \mathrm{~W} / \mathrm{m} \mathrm{K}$ at room temperature $)^{2,11}$ whose sintering parameters favored grain coarsening. Indeed, the sintering temperature was much higher in Refs. 2, 11, and 20 than that reported in Table I $\left(1450 \mathrm{~K},{ }^{2} 1523 \mathrm{~K}^{11}\right.$ and $\left.1473 \mathrm{~K}^{20}\right)$. Moreover, for hot pressing, the sintering time was intrinsically much longer.

\section{Theoretical thermal conductivity of $100 \%$ dense $\mathrm{CrSi}_{2}$}

The thermal conductivity of $100 \%$ theoretically dense nanostructured $\mathrm{CrSi}_{2}$ has been calculated by the extrapolation of the $94 \%$ and $74 \%$ dense sample through the well-known Eucken's
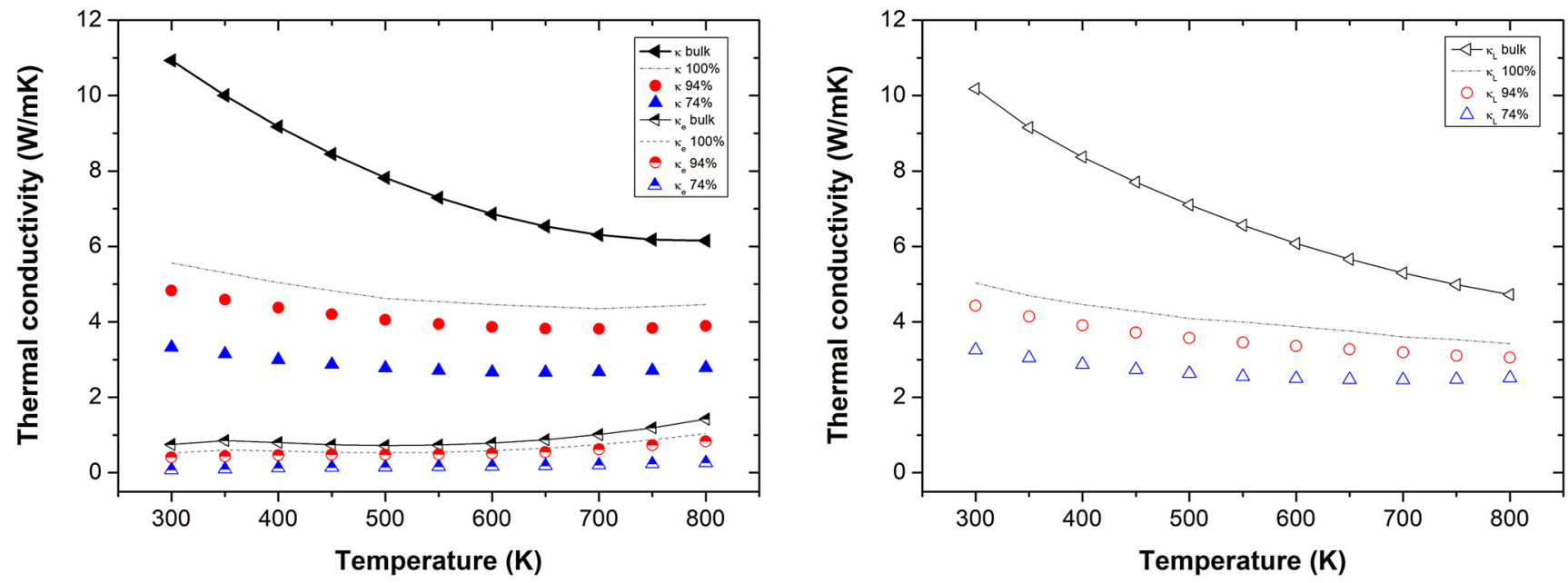

FIG. 5. Influence of the porosity on the total thermal conductivity $\kappa$ and electronic thermal conductivity $\kappa_{e}$ (left) and on the lattice contribution of the thermal conductivity $\kappa_{l}$ (right) of nano- $\mathrm{CrSi}_{2}$ alloys compared to bulk $\mathrm{CrSi}_{2}{ }_{2}^{2}$ and simulated $100 \%$ density-the $45 \mathrm{~nm}$ crystallite size sample. 
TABLE II. Fitted $A, B$, and $D$ parameters for the lattice thermal conductivity of bulk $\mathrm{CrSi}_{2}{ }^{2}$ and nano-CrSi 2 (45 nm) with a simulated density of $100 \%$ and with experimental samples with a density of $94 \%$ and $74 \%$.

\begin{tabular}{lcccc}
\hline \hline & Bulk $^{2}$ & $100 \%$ (sim.) & $94 \%$ (exp.) & $74 \%$ (exp.) \\
\hline$A\left(10^{-43} \mathrm{~s}^{3}\right)$ & 0.17 & 4.38 & 5.71 & 12.22 \\
$B\left(10^{-18} \mathrm{~s} / \mathrm{K}\right)$ & 3.64 & 3.64 & 3.64 & 3.64 \\
$D(\mathrm{~m})$ & $5.0010^{-6}$ & $4.5010^{-8}$ & $4.5010^{-8}$ & $4.5010^{-8}$ \\
Microstrain (\%) & $\ldots$ & $\ldots$ & 0.07 & 0.15 \\
$\mathrm{R}^{2}$ & 0.99 & 0.97 & 0.98 & 0.96 \\
\hline \hline
\end{tabular}

formula given below,

$$
\kappa_{\text {porous }}=\kappa_{\text {bulk }} \frac{2-2 \phi}{2+\phi},
$$

where $\kappa_{\text {porous }}$ and $\kappa_{\text {bulk }}$ are, respectively, the thermal conductivity of porous and dense samples and $\phi$ stands for the porosity. The thermal conductivity of the $100 \%$ theoretically dense sample obtained with this model is quite similar whether using the thermal conductivity obtained for the $94 \%$ or for the $74 \%$ dense sample. The averaged results are consequently given in Fig. 5 (black dashed lines) and give a prediction of the total thermal conductivity of $\sim 5.5 \mathrm{~W} / \mathrm{mK}(\sim 4.9 \mathrm{~W} / \mathrm{mK}$ for the lattice thermal conductivity) at RT.

\section{Callaway-Klemens model}

As the present report is the first one reporting detailed thermal variation of the thermal conductivity of $\mathrm{CrSi}_{2}$ with nanometric grain size, we now describe the impact of the small grain size on the thermal conductivity of $\mathrm{CrSi}_{2}$ compared to the other phonon scattering mechanisms already present in the bulk samples. To reach this goal, we have performed theoretical fits of the lattice thermal conductivity for our various samples, of the simulated data with $100 \%$ dense, and of the bulk sample from Daspgupta's work ${ }^{2}$ using
Klemens-Callaway's expression ${ }^{21-23}$

$$
\kappa_{l}(T)=\frac{k_{B}}{2 \pi^{2} v}\left(\frac{k_{B} T}{\hbar}\right)^{3} \int_{0}^{\theta_{D} / T} \frac{x^{4} e^{x}}{\tau_{C}^{-1}\left(e^{x}-1\right)^{2}} d x,
$$

where $x=\hbar \omega / k_{B} T$ is dimensionless, $\omega$ is the phonon frequency, $k_{B}$ is the Boltzmann constant, $\hbar$ is the reduced Planck constant, $\theta_{D}$ is the Debye temperature, $v$ is the sound velocity, and $\tau_{C}$ is the phonon scattering relaxation time. From room to high temperature, the effective phonon scattering relaxation rate $\tau_{C}^{-1}$ can be written as ${ }^{24}$

$$
\tau_{C}^{-1}=\tau_{M}^{-1}+\tau_{U}^{-1}+\tau_{B}^{-1}
$$

The relaxation rate for the defect/mass-difference scattering is given by the following expression:

$$
\tau_{M}^{-1}=\frac{V_{0} \Gamma \omega^{4}}{4 \pi v^{3}}
$$

where $\Gamma$ is the impurity scattering parameter and $V_{0}$ is the volume per atom.

The relaxation rate for Umklapp scattering at high temperature (room and above) can be taken as the one given by Klemens as ${ }^{23}$

$$
\tau_{U}^{-1}=\frac{2 \gamma^{2} k_{B} T \omega^{2}}{\mu V_{0} \omega_{D}}
$$

where $\gamma$ is the Gruneisen anharmonicity parameter, $\mu$ is the shear modulus, and $\omega_{D}$ is the Debye frequency. The shear modulus in Eq. (5) is treated as a velocity dependent effective value calculated for a given geometry.

The boundary scattering mechanism is particularly important for low-dimensional nanostructures and its relaxation time is
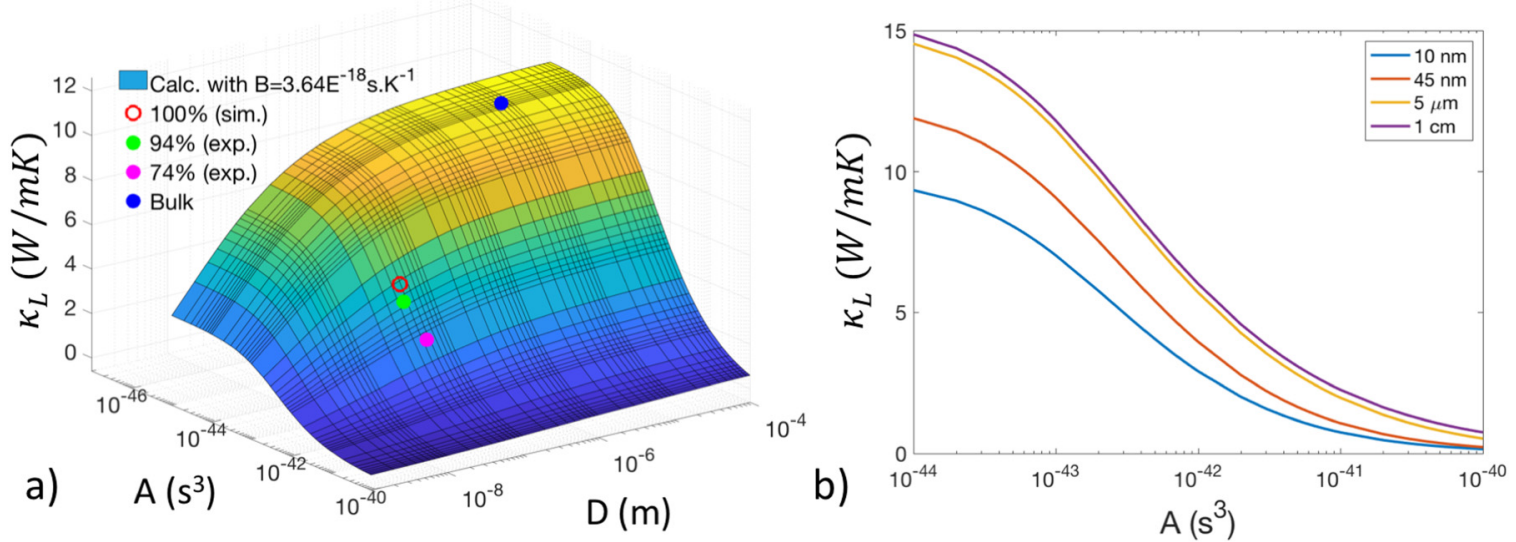

FIG. 6. Lattice thermal conductivity simulated with Klemens-Callaway expressions (2)-(8) at $300 \mathrm{~K}$ with $B=3.64 \quad 10^{-18} \mathrm{~s} / \mathrm{K}$ (left) as a function of $A$ and $D$ and (right) as a function of $A$ with $D$ equal to $10 \mathrm{~nm}, 45 \mathrm{~nm}, 5 \mu \mathrm{m}$, and $1 \mathrm{~cm}$. Fitted parameters reported in Table II (Bulk' $100 \%, 94 \%$, and $74 \%$ ) are also located on the map (left). 
given by

$$
\tau_{B}^{-1}=\frac{v}{D},
$$

where $D$ is the dimension of the system. By combining Eqs. (3)-(6), the phonon scattering relaxation rate can be written as

$$
\tau_{C}^{-1}=\frac{V_{0} \Gamma \omega^{4}}{4 \pi v^{3}}+\frac{2 \gamma^{2} k_{B} T \omega^{2}}{\mu V_{0} \omega_{D}}+\frac{v}{D}
$$
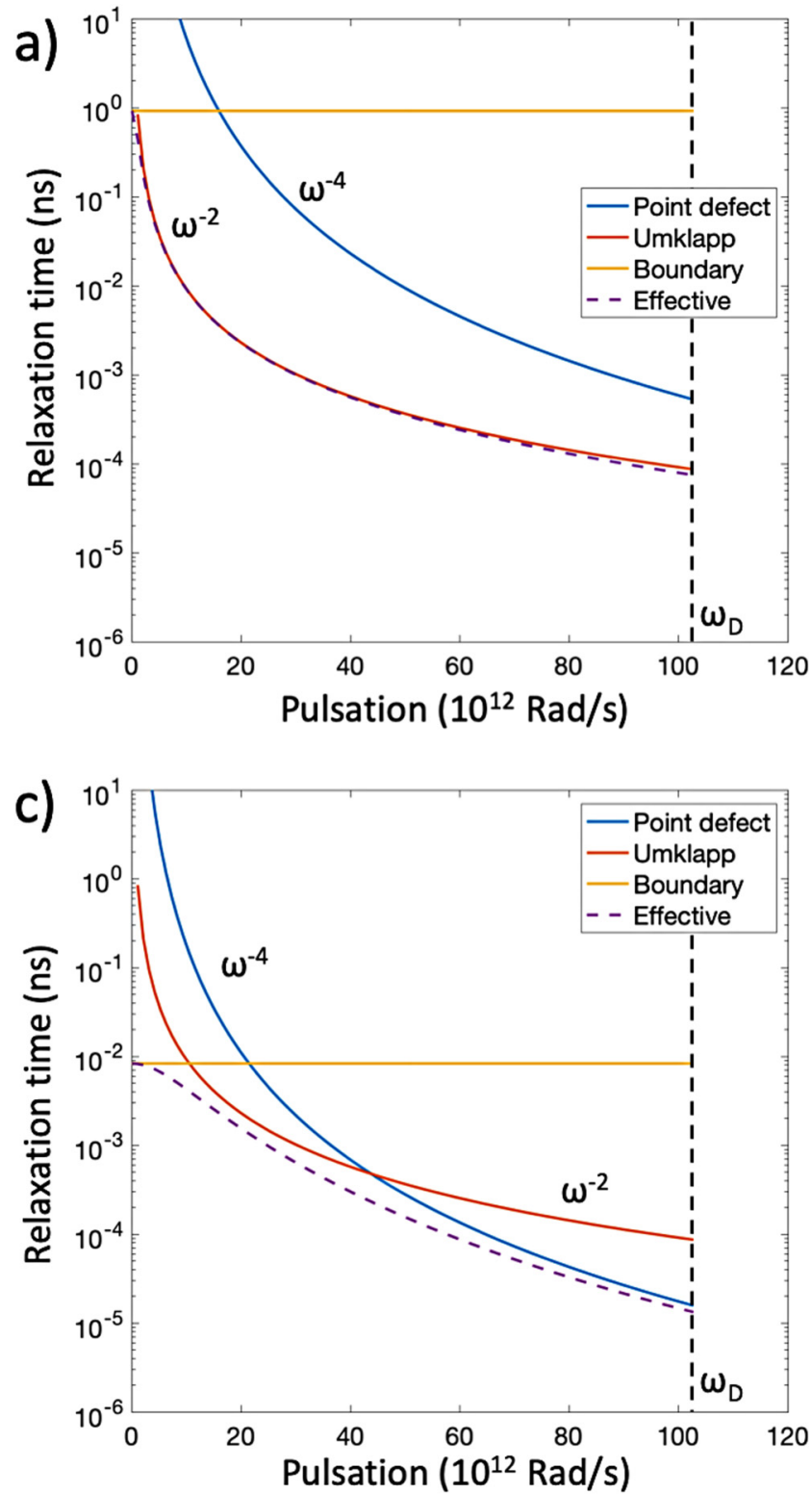

In order to simplify the problem, we introduce the parameters $A$ and $B$ such as

$$
\tau_{C}^{-1}=A \omega^{4}+B \omega^{2} T+\frac{v}{D} .
$$

The fitting parameters are listed in Table II. To limit the number of fitted parameters,

- We fixed the prefactor $B$ for the phonon-phonon Umklapp scattering for all the samples to the value $B=3.6410^{-18} \mathrm{~s} / \mathrm{K}$, after
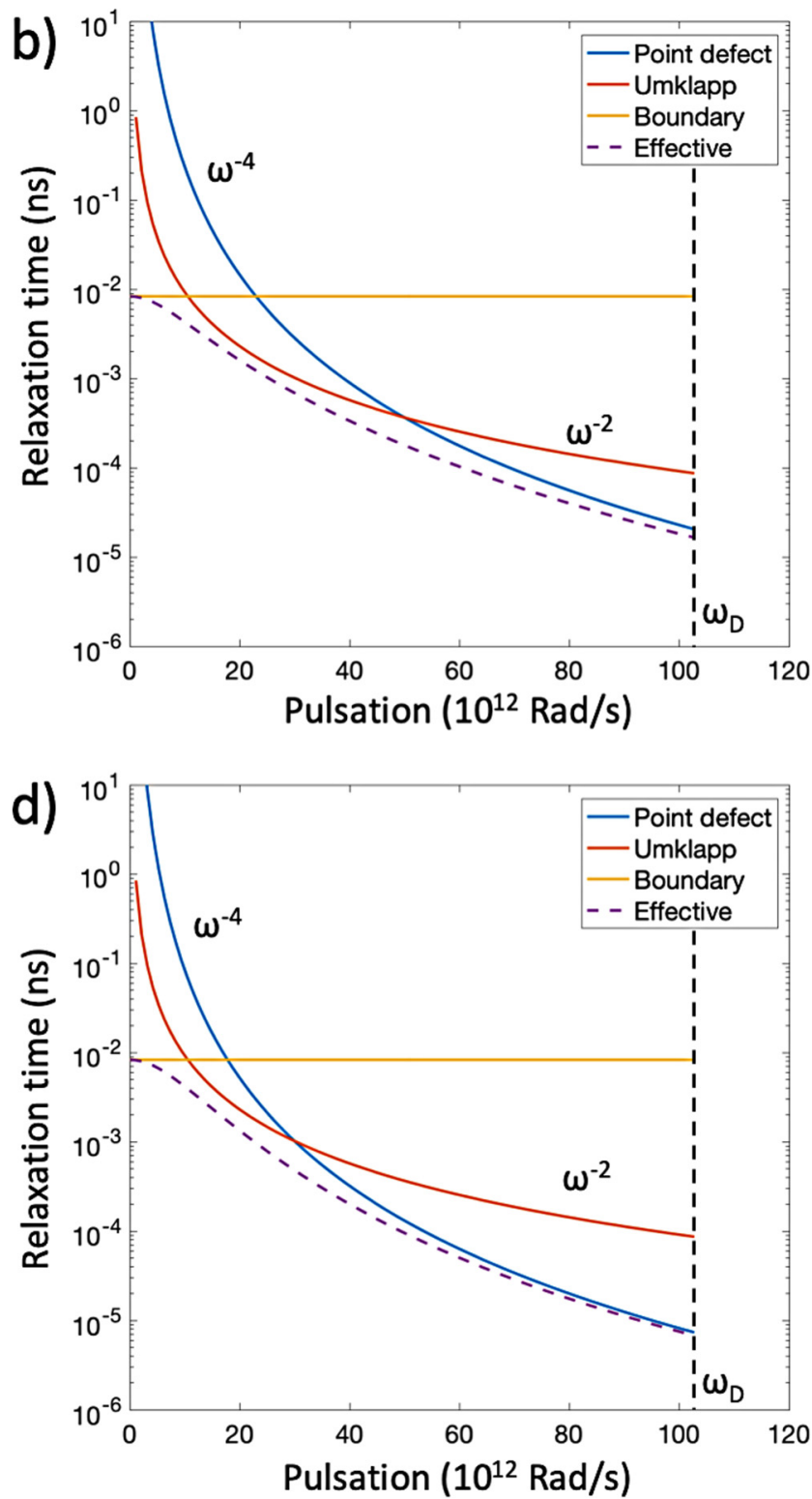

FIG. 7. Effective relaxation time and relaxation time for point defects, Umklapp and boundary scattering mechanism for (a) Bulk, ${ }^{2}$ (b) $100 \%$ (sim.), (c) $94 \%$ (exp.), and (d) $74 \%$ (exp.) dense samples at $300 \mathrm{~K}$. The Debye pulsation $\omega_{D}$ has been calculated using the experimental Debye temperature of $793 \mathrm{~K}$. 
fitting the $94 \%$ dense sample. Indeed, this parameter is intrinsic to $\mathrm{CrSi}_{2}$ and should not be affected by the grain size reduction.

- The grain size $D$ is determined experimentally to $\sim 45 \mathrm{~nm}$, as seen above, for the $94 \%$ and $74 \%$ samples. For the bulk sample, based on the metallurgical process used by Dasgupta et $a .^{2}$ and our experience in the synthesis of this alloy, we assume that $D$ is about a micrometer or even larger. For such a dimension, it does not influence much the relaxation rate for the mass-difference scattering parameter $A$ as seen in Fig. 6.

The theoretical model fits the experimental data very well in the temperature range $300 \mathrm{~K}-600 \mathrm{~K}$ (see Table II and Fig. S4 in the supplementary material). A small deviation from the experimental data was observed (not shown here) at higher temperatures, which may be due to the bipolar contribution. We also observe a small effect of the phonon scattering by the point defects in Dasgupta's bulk sample, whereas it increases strongly in the nanostructured samples. This must be due to the ball milling process, which increases significantly the defect density in our samples. Likewise, one notices that the defect/mass-difference scattering parameter's $A$ is smaller for the $94 \%$ than for $74 \%$ nanostructured samples, in agreement with a smaller internal residual strain which is about $0.07 \%$ and $0.15 \%$, respectively. This behavior can be also related to the larger sintering temperature for the $94 \%$ dense sample compared to the $74 \%$ dense sample. Therefore, $t$ mechanical milling has two effects for decreasing the lattice thermal conductivity: by increasing the grain boundary scattering and by increasing the defect scattering. As shown in Fig. 6 (right), with a grain size of $45 \mathrm{~nm}$, an increase of the defect/mass-difference scattering could lead to a stronger decrease of the thermal conductivity, which is consistent with Eq. (5).

As shown in Fig. 7 and as expected, the boundary scattering mechanism is predominant at low pulsation $\omega$ for nanostructured samples and negligible for bulk samples. One also notices that the transition from an Umklapp scattering mechanism to point defect scattering mechanism predominance shifts to low frequency when the porosity increases. This behavior is consistent with the increase of defects observed when the porosity increases (see Table II).

\section{CONCLUSION}

In the present study, we show that it is possible to obtain nanostructured $\mathrm{CrSi}_{2}$ samples with about $10 \mathrm{~nm}$ grain size after a few hours of ball milling of the bulk $\mathrm{CrSi}_{2}$ sample. We observe that the nanostructured $\mathrm{CrSi}_{2}$ samples are fairly stable up to $1073 \mathrm{~K}$ and that strong grain coarsening occurs beyond this temperature. This means that for avoiding grain coarsening, the temperature range of use must remain under $1073 \mathrm{~K}$. In order to limit the grain coarsening while densifying the powders in the shape of pellets, we have used the SPS technique. We were able to obtain pellet with high density (94\%) and keep a small grain size of about $45 \mathrm{~nm}$ diameter, which lead to a thermal conductivity of $\sim 5 \mathrm{~W} / \mathrm{mK}$. We also predict that the $100 \%$ dense sample with the same microstructure would display a thermal conductivity of $\sim 5.5 \mathrm{~W} / \mathrm{mK}$, which is twice smaller than in bulk polycrystalline $\mathrm{CrSi}_{2}$ samples. We have analyzed the impact of the porosity, of the small grain size, and of the other phonon scattering mechanisms on the lattice thermal conductivity of the $\mathrm{CrSi}_{2}$ nanostructured samples. We have identified a large phonon scattering by the defects in both bulk polycrystalline and nanostructured $\mathrm{CrSi}_{2}$ samples. The large decrease of the lattice thermal conductivity in the $\mathrm{CrSi}_{2}$ nanostructured samples was shown to be due to both phonons scattering by the defects and by the grain boundaries.

\section{SUPPLEMENTARY MATERIAL}

See the supplementary material for the diffractogram of ballmilled $\mathrm{CrSi}_{2}$, of SPS pellets ( $74 \%$ and $94 \%$ ), the electrical resistivity, and the fitting of the thermal conductivity with the CallawayKlemens model.

\section{ACKNOWLEDGMENTS}

The authors wish to thank B. Rebière (IEM, Montpellier) for SEM and the University of Montpellier and the CheMISyst labex (No. ANR-10-LABX-05-01) for their funding.

\section{REFERENCES}

${ }^{1}$ M. I. Fedorov and G. N. Isachenko, Jpn. J. Appl. Phys. 54, 07JA05 (2015).

${ }^{2}$ T. Dasgupta, J. Etourneau, B. Chevalier, S. F. Matar, and A. M. Umarji, J. Appl. Phys. 103, 113516 (2008).

${ }^{3}$ I. Nishida and T. Sakata, J. Phys. Chem. Solids 39, 499 (1978).

${ }^{4}$ D. Shinoda, S. Asanabe, and Y. Sasaki, J. Phys. Soc. Jpn. 19, 269 (1964).

5I. Nishida, J. Mater. Sci. 7, 1119 (1972).

${ }^{6}$ T. Dasgupta and A. M. Umarji, J. Alloys Compd. 461, 292 (2008).

${ }^{7}$ P. Hermet, M. Khalil, R. Viennois, M. Beaudhuin, D. Bourgogne, and D. Ravot, RSC Adv. 5, 19106 (2015).

${ }^{8} \mathrm{M}$. Khalil, "Investigation and optimization of semiconducting chromium disilicide based materials for thermoelectric applications," Ph.D. thesis (Univ. Montpellier, Montpellier, 2015).

${ }^{9}$ V. K. Zaitsev, M. I. Fedorov, E. A. Gurieva, I. S. Eremin, P. P. Konstantinov, A. Y. Samunin, and M. V. Vedernikov, Phys. Rev. B 74, 45207 (2006).

${ }^{10}$ S. Karuppaiah, M. Beaudhuin, and R. Viennois, J. Solid State Chem. 199, 90 (2013).

${ }^{11}$ S. Perumal, S. Gorsse, U. Ail, M. Prakasam, B. Chevalier, and A. M. Umarji, I. Mater. Sci. 48, 6018 (2013).

${ }^{12}$ M. Khalil, M. Beaudhuin, B. Villeroy, D. Ravot, and R. Viennois, J. Alloys Compd. 662, 150 (2016).

${ }^{13}$ D. M. Rowe, Thermoelectric Handbook-Macro to Nano (CRC Press, 2006).

${ }^{14}$ B. K. Voronov, L. D. Dudkin, and N. N. Trusova, Sov. Powder Metall. Met. Ceram. 13, 962 (1974).

${ }^{15}$ J. Rodriguez-Carvajal, Satellite Meeting on Powder Diffraction of the XV Congress IUCr 127, Toulouse, France (Congress International Union of Crystallography, Toulouse, France, 1990).

${ }^{16}$ H. Chen, Y. Du, and J. C. Schuster, Calphad 33, 211 (2009).

${ }^{17}$ E. Alleno, D. Berardan, C. Byl, C. Candolfi, R. Daou, R. Decourt, E. Guilmeau, S. Hebert, J. Hejtmanek, B. Lenoir, P. Masschelein, V. Ohorodnichuk, M. Pollet, S. Populoh, D. Ravot, O. Rouleau, and M. Soulier, Rev. Sci. Instrum. 86, 011301 (2015)

${ }^{18}$ O. M. Lemine, Superlattices Microstruct. 45, 576 (2009).

${ }^{19}$ R. Yazdani-rad, S. A. Mirvakili, and M. Zakeri, J. Alloys Compd. 489, 379 (2010).

${ }^{20}$ A. Mohamad, Y. Ohishi, Y. Miyazaki, H. Muta, K. Kurosaki, and S. Yamanaka, Mater. Trans. 57, 1059 (2016).

${ }^{21}$ J. Callaway, Phys. Rev. 113, 1046 (1959).

${ }^{22}$ C. T. Walker and R. O. Pohl, Phys. Rev. 131, 1433 (1963).

${ }^{23}$ P. G. Klemens, Solid State Physics (Academic Press Inc., NY, 1958), Vol. 7.

${ }^{24}$ K. S. Dubey and R. H. Misho, Phys. Status Solidi 84, 69 (1977).

${ }^{25}$ M. Nakamura, Metall. Mater. Trans. A 25, 331 (1994). 